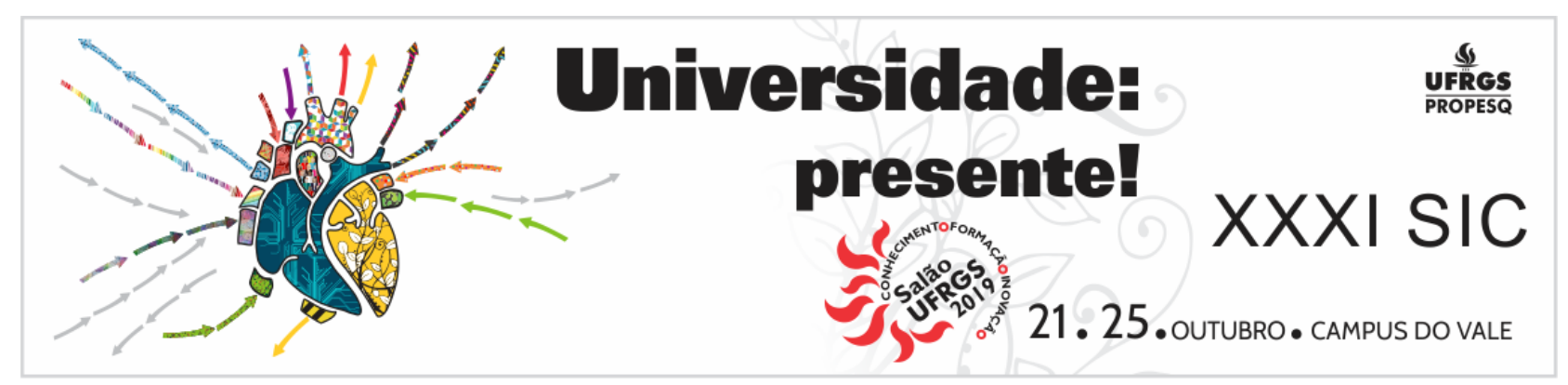

\begin{tabular}{|l|l|}
\hline Evento & $\begin{array}{l}\text { Salão UFRGS 2019: SIC - XXXI SALÃO DE INICIAÇÃO } \\
\text { CIENTÍFICA DA UFRGS }\end{array}$ \\
\hline Ano & 2019 \\
\hline Local & Campus do Vale - UFRGS \\
\hline Título & $\begin{array}{l}\text { Non-intrusive Fault Injection Techniques for Efficient Soft Error } \\
\text { Vulnerability Analysis }\end{array}$ \\
\hline Autor & VITOR VIANA BANDEIRA \\
\hline Orientador & RICARDO AUGUSTO DA LUZ REIS \\
\hline
\end{tabular}




\title{
Non-intrusive Fault Injection Techniques for Efficient Soft Error Vulnerability Analysis
}

\author{
Vitor Viana Bandeira (Bolsista IC) \\ Prof. Dr. Ricardo A. L. Reis (Orientador) \\ \{vvbandeira,reis\}@inf.ufrgs.br
}

21 de junho de 2019

\section{Resumo}

Sistemas eletrônicos estão integrando processadores multicore e GPUs (Graphical Processing Unit) com o objetivo de executar configurações complexas de aplicações. É esperado que esses sistemas sofram com pelo menos uma falha por dia nos próximos anos, o que pode levar a erros que coloquem a vida de outros em perigo. Esse trabalho apresenta quatro novas técnicas de injeção de falhas que possibilitam um maior controle e analise do comportamento de sistemas multicore. Essas técnicas são não intrusivas, isto é, não precisamos modificar o software ou o hardware, e são integradas em um framework para execução automática. Para validá-las, nos utilizamos aplicações reais com ate 43 bilhões de instruções. Resultados inicias mostram que isolar partes criticas de uma aplicação pode levar a uma analise de soft-error mais eficiente, ou seja, reduzir o numero de falhas mascaradas em até $28 \%$. Falhas mascaradas não proveem informação sobre o que aconteceria com o sistema, mas sim a probabilidade de um erro ocorrer.

\section{Abstract}

Electronic computing systems are integrating modern multicore processors and GPUs aiming to perform complex software stacks in different life-critical systems, including health devices and emerging self-driving cars. Such systems are expected to experience at least one soft error per day in the near future, which may lead to life-threatening failures. This paper presents four novel non-intrusive fault injection techniques that enable full fault injection control and inspection of multicore systems behavior in the presence of faults. Proposed techniques were integrated into a fault injection framework and verified through a real automotive case study with up to 43 billions instructions. Results show that isolating critical application functions leads to a more efficient soft error analysis, i.e., reduction of masked faults in up to $28 \%$. 